\title{
THERMOELASTIC WAVES WITHOUT ENERGY DISSIPATION IN AN UNBOUNDED BODY WITH A SPHERICAL CAVITY
}

\author{
D. S. CHANDRASEKHARAIAH and K. S. SRINATH
}

(Received 20 March 1998)

\begin{abstract}
The linear theory of thermoelasticity without energy dissipation is employed to study waves emanating from the boundary of a spherical cavity in a homogeneous and isotropic unbounded thermoelastic body. The waves are supposed to be spherically symmetric and caused by a constant step in temperature applied to the stress-free boundary of the cavity. Small-time solutions for the displacement, temperature, and stress fields are obtained by using the Laplace transform technique. It is found that there exist two coupled waves, of which one is predominantly elastic and the other is predominantly thermal, both propagating with finite speeds but with no exponential attenuation. Exact expressions for discontinuities in the field functions that occur at the wavefronts are computed and analysed. The results are compared with those obtained earlier in the contexts of some other models of thermoelasticity.
\end{abstract}

Keywords and phrases. Thermoelasticity without energy dissipation, generalized thermoelasticity, thermoelastic spherical waves.

2000 Mathematics Subject Classification. Primary 74H99, 74S30, 74J10.

1. Introduction. Thermoelasticity theories that admit finite speeds for thermal signals have aroused much interest in the last three decades. In contrast to the conventional coupled thermoelasticity theory based on a parabolic heat equation [1], which predicts an infinite speed for the propagation of heat, these theories involve hyperbolic heat equations and are referred to as generalized thermoelasticity theories. For details about the physical relevance of these theories and a review of the relevant literature, see [2].

Recently, Green and Naghdi [14] formulated a new generalized thermoelasticity theory by including the so-called "thermal-displacement gradient" among the independent constitutive variables. An important characteristic feature of this theory, which is not present in other thermoelasticity theories, is that this theory does not accommodate dissipation of thermal energy. In the context of the linearized version of this theory, theorems on uniqueness of solutions have been established in [3, 6], boundaryinitiated waves in a half-space and in unbounded body with cylindrical cavity have been studied in $[4,9,10,11]$, and plane harmonic waves and Rayleigh waves have been studied in $[5,7,12]$.

In this paper, we employ the theory formulated in [14] (hereafter referred to as the GN-theory) to study spherically symmetric thermoelastic waves in a linear, homogeneous and isotropic unbounded solid body containing a spherical cavity. We suppose that the waves are generated due to the application of a constant step in 
temperature on the boundary of the cavity which is held in a stress-free state. We employ the Laplace transform technique to analyse the problem. We restrict ourselves to the derivation of small-time solutions for the field functions. We find that there occur two coupled waves both propagating with finite speeds, of which one is predominantly elastic and the other is predominantly thermal, and that these waves do not experience any attenuation. We further find that the displacement field is continuous whereas the thermal and stress fields are discontinuous at both the wavefronts. We obtain exact expressions for the discontinuities and make a brief analysis thereof.

The counterparts of our problem in the contexts of the uncoupled thermoelasticity theory, the coupled thermoelasticity theory, the Green-Lindsay theory (GL-theory) [13] and the Lord-Shulman theory (LS-theory) [15] have been considered in [16, 17, 18, 20], respectively. At appropriate stages of our analysis, we make a comparison of our results with those obtained in these works. This comparison reveals that, on the whole, the predictions of the GN-theory (as obtained here) are qualitatively similar to those of the LS-theory. More importantly, we notice that certain physically unrealistic features inherent in the conventional coupled thermoelasticity theory and the GL-theory are not present in the GN-theory.

2. Formulation of the problem. In the context of the GN-theory, the field equations for a linear, homogeneous and isotropic thermoelastic solid body, in the absence of body forces and heat sources, are as follows [14]:

$$
\begin{aligned}
& \mu \nabla^{2} \mathbf{u}+(\lambda+\mu) \nabla \operatorname{div} \mathbf{u}-\gamma \nabla \theta=\rho \ddot{\mathbf{u}}, \\
& c \ddot{\theta}+\gamma T_{0} \operatorname{div} \ddot{\mathbf{u}}=\kappa^{*} \nabla^{2} \theta .
\end{aligned}
$$

In these equations, $\mathbf{u}$ is the displacement vector; $\theta$ is the temperature-change above a uniform reference temperature $T_{0} ; \rho$ is the mass density; $c$ is the specific heat; $\lambda$ and $\mu$ are the Lame' constants; $\gamma=(3 \lambda+2 \mu) \beta^{*}, \beta^{*}$ being the coefficient of volume expansion; and $\kappa^{*}$ is a material constant characteristic of the theory.

The stress tensor $\mathbf{T}$ associated with $\mathbf{u}$ and $\theta$ is given by [14]

$$
\mathbf{T}=\lambda(\operatorname{div} \mathbf{u}) \mathbf{I}+\mu\left(\nabla \mathbf{u}+\nabla \mathbf{u}^{T}\right)-\gamma \theta \mathbf{I} .
$$

In all the above equations, the direct vector/tensor notation [8] is employed. Also, an over dot denotes partial derivative with respect to the time variable $t$. Some of our symbols and the notation are slightly different from those employed in [14].

For spherically symmetric interactions, the displacement vector possesses only the radial component $u=u(r, t)$, where $r$ is the radial distance measured from the origin (point of symmetry), and the stress tensor is determined by the radial stress $\sigma_{r}$ and the circumferential stress (hoop stress) $\sigma_{\phi}$. In this case, equations (2.1) yield the following governing equations for $u$ and $\theta$ :

$$
\begin{gathered}
(\lambda+2 \mu)\left[\frac{\partial}{\partial r}\left(\frac{\partial u}{\partial r}+\frac{2}{r} u\right)\right]-\gamma \frac{\partial \theta}{\partial r}=\rho \frac{\partial^{2} u}{\partial t^{2}}, \\
c \frac{\partial^{2} \theta}{\partial t^{2}}+\gamma T_{0} \frac{\partial^{2}}{\partial t^{2}}\left(\frac{\partial}{\partial r}+\frac{2}{r}\right) u=\kappa^{*}\left(\frac{\partial^{2}}{\partial r^{2}}+\frac{2}{r} \frac{\partial}{\partial r}\right) \theta .
\end{gathered}
$$


Also, the relation (2.2) yields

$$
\begin{aligned}
& \sigma_{r}=(\lambda+2 \mu) \frac{\partial u}{\partial r}+2 \lambda \frac{u}{r}-\gamma \theta, \\
& \sigma_{\phi}=\lambda \frac{\partial u}{\partial r}+2(\lambda+\mu) \frac{u}{r}-\gamma \theta .
\end{aligned}
$$

For a mathematical analysis, it is convenient to have (2.3) and (2.4) rewritten in non-dimensional form. For this purpose, we consider the transformations

$$
\begin{gathered}
r^{\prime}=\frac{r}{L}, \quad t^{\prime}=\frac{V}{L} t, \quad u^{\prime}=\frac{(\lambda+2 \mu)}{L \gamma T_{0}} u, \\
\theta^{\prime}=\frac{\theta}{T_{0}}, \quad \sigma_{r}^{\prime}=\frac{\sigma_{r}}{\gamma T_{0}}, \quad \sigma_{\phi}^{\prime}=\frac{\sigma_{\phi}}{\gamma T_{0}},
\end{gathered}
$$

where $L$ is a standard length and $V$ is a standard speed. Using these transformations in (2.3) and (2.4) and suppressing primes for simplicity in the notation, we obtain the following equations/relations which are in non-dimensional form:

$$
\begin{gathered}
C_{p}^{2}\left[\frac{\partial}{\partial r}\left(\frac{\partial u}{\partial r}+\frac{2}{r} u\right)-\frac{\partial \theta}{\partial r}\right]=\frac{\partial^{2} u}{\partial t^{2}}, \\
C_{T}^{2}\left(\frac{\partial^{2} \theta}{\partial r^{2}}+\frac{2}{r} \frac{\partial \theta}{\partial r}\right)=\frac{\partial^{2} \theta}{\partial t^{2}}+\varepsilon \frac{\partial^{2}}{\partial t^{2}}\left(\frac{\partial u}{\partial r}+\frac{2}{r} u\right), \\
\sigma_{r}=\frac{\partial u}{\partial r}+2 \eta \frac{u}{r}-\theta \\
\sigma_{\phi}=\eta \frac{\partial u}{\partial r}+(1+\eta) \frac{u}{r}-\theta .
\end{gathered}
$$

Here,

$$
C_{P}^{2}=\frac{(\lambda+2 \mu)}{\rho V^{2}}, \quad C_{T}^{2}=\frac{\kappa^{*}}{c V^{2}}, \quad \varepsilon=\frac{\gamma^{2} T_{0}}{c(\lambda+2 \mu)}, \quad \eta=\frac{\lambda}{\lambda+2 \mu} .
$$

We note that $C_{P}$ and $C_{T}$ respectively represent the non-dimensional speeds of purely elastic dilatational wave and purely thermal wave, and $\varepsilon$ is the usual thermoelastic coupling factor.

The body being considered for our analysis here is an unbounded thermoelastic solid with a spherical cavity. We choose the origin at the centre of the cavity and denote the dimensionless radius of the cavity by $a$. If initially the body is at rest in an undeformed state and has its temperature-change and temperature-rate equal to zero, then the following initial conditions hold:

$$
u=\frac{\partial u}{\partial t}=\theta=\frac{\partial \theta}{\partial t}=0 \quad \text { at } t=0 \text { for } r \geq a
$$

If the thermoelastic interactions are caused by a uniform step in temperature applied to the boundary of the cavity which is held in the stress-free state, then the following boundary conditions hold:

$$
\theta=\chi H(t), \quad \sigma_{r}=0 \quad \text { for } r=a, t>0,
$$


where $\mathcal{X}$ is a positive constant and $H(t)$ is the Heaviside unit step function. With the aid of (2.7), these boundary conditions can be rewritten as

$$
\frac{\partial u}{\partial r}+2 \eta \frac{u}{r}=\chi H(t) \text { for } r=a, t>0 .
$$

Thus, for our problem here, (2.6) are the governing differential equations, (2.10) are the initial conditions, and (2.12) is the boundary condition. Once $u$ and $\theta$ are determined by solving this initial-boundary value problem, then $\sigma_{r}$ and $\sigma_{\phi}$ can be computed by using (2.7) and (2.8).

3. Transform solution. Taking the Laplace transforms of (2.6) under the homogeneous initial conditions (2.10), we obtain the following equations:

$$
\begin{aligned}
& {\left[C_{p}^{2} D D_{1}-s^{2}\right] \bar{u}=C_{P}^{2} D \bar{\theta},} \\
& {\left[C_{T}^{2} D_{1} D-s^{2}\right] \bar{\theta}=\varepsilon s^{2} D_{1} \bar{u} .}
\end{aligned}
$$

Here, an over bar denotes the Laplace transform of the corresponding function, $s$ is the transform parameter, and

$$
D=\frac{d}{d r}, \quad D_{1}=D+\frac{2}{r} .
$$

The coupled (3.1) and (3.2) can be decoupled by eliminating $\bar{\theta}$ or $\bar{u}$, and put in the form

$$
\begin{aligned}
& \left(D D_{1}-m_{1}^{2}\right)\left(D D_{1}-m_{2}^{2}\right) \bar{u}=0, \\
& \left(D_{1} D-m_{1}^{2}\right)\left(D_{1} D-m_{2}^{2}\right) \bar{\theta}=0,
\end{aligned}
$$

where $m_{1}^{2}$ and $m_{2}^{2}$ satisfy the quadratic equation

$$
C_{p}^{2} C_{T}^{2} x^{2}-\left[C_{T}^{2}+(1+\varepsilon) C_{P}^{2}\right] s^{2} x+s^{4}=0 .
$$

Under the regularity conditions that $\bar{u}$ and $\bar{\theta} \rightarrow 0$ as $r \rightarrow \infty$, the general solutions of (3.4) are given by

$$
\begin{aligned}
& \bar{u}=\sum_{\alpha=1}^{2} A_{\alpha}\left(\frac{1}{r}+\frac{1}{m_{\alpha} r^{2}}\right) e^{-m_{\alpha} r}, \\
& \bar{\theta}=\frac{1}{r} \sum_{\alpha=1}^{2} B_{\alpha} e^{-m_{\alpha} r}
\end{aligned}
$$

where $A_{\alpha}$ and $B_{\alpha}, \alpha=1,2$, are functions of $s$, of which only $A_{\alpha}$ or $B_{\alpha}$ are arbitrary, and $m_{\alpha}$ is the square root, with positive real part, of $m_{\alpha}^{2}$. By solving (3.5), we obtain the following exact expressions for $m_{\alpha}$ :

$$
m_{\alpha}=\frac{s}{V_{\alpha}},
$$

where

$$
V_{\alpha}=\frac{1}{\sqrt{2}}\left[C_{T}^{2}+(1+\varepsilon) C_{P}^{2}+(-1)^{\alpha+1} \Delta\right]^{1 / 2}
$$


with

$$
\Delta=\left\{\left[C_{T}^{2}-(1+\varepsilon) C_{P}^{2}\right]^{2}+4 \varepsilon C_{P}^{2} C_{T}^{2}\right\}^{1 / 2} .
$$

Here and in the expressions that follow, the suffix $\alpha$ takes values 1 and 2 .

Substituting for $\bar{u}$ and $\bar{\theta}$ from (3.6) and (3.7) in (3.2) and equating the corresponding coefficients, we get

$$
B_{\alpha}=\frac{\varepsilon s^{2} m_{\alpha}}{s^{2}-C_{T}^{2} m_{\alpha}^{2}} A_{\alpha} .
$$

Next, taking the Laplace transform of the boundary condition (2.12) and substituting for $\bar{u}$ from (3.6) in the resulting expression, we obtain

$$
A_{\alpha}=(-1)^{\alpha+1} \frac{\Omega_{\alpha}}{\Gamma},
$$

where

$$
\begin{aligned}
\Omega_{\alpha} & =\frac{a \chi}{m_{\alpha}}\left(C_{T}^{2} m_{\alpha}^{2}-s^{2}\right)\left[\varepsilon s^{2} a^{2} m_{1}^{2} m_{2}^{2}-\left(C_{T}^{2} m_{3-\alpha}^{2}-s^{2}\right) \Lambda_{\alpha}\right] e_{\alpha a}^{m}, \\
\Gamma & =\varepsilon s^{3}\left[\left(C_{T}^{2} m_{2}^{2}-s^{2}\right) \Lambda_{1}-\left(C_{T}^{2} m_{1}^{2}-s^{2}\right) \Lambda_{2}\right], \\
\Lambda_{\alpha} & =m_{\alpha}^{2}\left[m_{3-\alpha}^{2} a^{2}+2(1-\eta) m_{3-\alpha} a+2(1-\eta)\right] .
\end{aligned}
$$

Substituting for $m_{\alpha}, A_{\alpha}$ and $B_{\alpha}$ from (3.8) and (3.11), (3.12), and (3.13) in (3.6) and (3.7), we obtain expressions for $\bar{u}$ and $\bar{\theta}$ in terms of $r$ and $s$. The forms of these expressions indicate that each of the displacement and thermal fields (and consequently the stress field) consists of two parts and that each part corresponds to a wave propagating with a finite speed, the wave corresponding to the first part having its speed equal to $V_{1}$ and that corresponding to the second part equal to $V_{2}$. We find that the expressions giving these speeds, namely, (3.9) and (3.10), are identical with the expressions giving the speeds of boundary-initiated plane waves in a half-space [4]. As in [4], it can be shown that $V_{1}>V_{2}$ and that the faster wave is predominantly elastic or predominantly thermal and the slower wave is predominantly thermal or predominantly elastic according as $C_{P}>C_{T}$ or $C_{T}>C_{P}$.

4. Small-time solution. We notice that $A_{\alpha}$, given by (3.12) and (3.13), and therefore $B_{\alpha}$, given by (3.11), are complicated functions of $s$. Consequently, the Laplace transform inversion of the expressions (3.6) and (3.7) for all $s$ is a formidable task. We confine ourselves to the derivation of small-time solutions for the field functions. This is done by taking $s$ to be large (as in $[16,17,18]$, for example).

When $s$ is large, the results (3.8) and (3.11), (3.12), and (3.13) yield the following approximate expressions for $A_{\alpha}$ and $B_{\alpha}$ (after some straightforward but lengthy calculations):

$$
\begin{aligned}
& A_{\alpha} \approx(-1)^{\alpha+1} \chi V_{\alpha}\left[\frac{L_{\alpha}}{s^{2}}+\frac{M_{\alpha}}{s^{3}}\right] \exp \left(\frac{s a}{V_{\alpha}}\right), \\
& B_{\alpha} \approx(-1)^{\alpha+1} \chi\left[\frac{N_{\alpha}}{s}+\frac{P_{\alpha}}{s^{2}}\right] \exp \left(\frac{s a}{V_{\alpha}}\right),
\end{aligned}
$$


where

$$
\begin{gathered}
L_{\alpha}=\frac{a}{\varepsilon C_{T}^{2}\left(V_{1}^{2}-V_{2}^{2}\right)}\left(V_{\alpha}^{2}-C_{T}^{2}\right)\left[C_{T}^{2}-(1+\varepsilon) V_{3-\alpha}^{2}\right], \\
M_{\alpha}=\frac{2(1-\eta)}{\varepsilon C_{T}^{2}\left(V_{1}^{2}-V_{2}^{2}\right)}\left(C_{T}^{2}-V_{\alpha}^{2}\right)\left\{V_{3-\alpha}\left(V_{3-\alpha}^{2}-C_{T}^{2}\right)-\frac{\left(V_{1} V_{2}+C_{T}^{2}\right)}{C_{T}^{2}\left(V_{1}+V_{2}\right)}\left[(1+\varepsilon) V_{3-\alpha}^{2}-C_{T}^{2}\right]\right\}, \\
\left(N_{\alpha}, P_{\alpha}\right)=\frac{\varepsilon V_{\alpha}^{2}}{\left(V_{\alpha}^{2}-C_{T}^{2}\right)}\left(L_{\alpha}, M_{\alpha}\right) .
\end{gathered}
$$

Now, using (3.8) and (4.1) in (3.6) and (3.7), and inverting the resulting expressions with the aid of standard formulas of the Laplace transform theory [19], we obtain the following solutions for $u$ and $\theta$ valid for small values of $t$ :

$$
\begin{aligned}
& u=\frac{\chi}{r} \sum_{\alpha=1}^{2}(-1)^{\alpha+1} V_{\alpha}\left[L_{\alpha} \tau_{\alpha}+\frac{1}{2}\left(\frac{1}{r} V_{\alpha} L_{\alpha}+M_{\alpha}\right) \tau_{\alpha}^{2}\right] H\left(\tau_{\alpha}\right), \\
& \theta=\frac{\chi}{r} \sum_{\alpha=1}^{2}(-1)^{\alpha+1}\left(N_{\alpha}+P_{\alpha} \tau_{\alpha}\right) H\left(\tau_{\alpha}\right) .
\end{aligned}
$$

Here,

$$
\tau_{\alpha}=\left(t-\frac{r-a}{V_{\alpha}}\right)
$$

Substituting the above solutions for $u$ and $\theta$ in the relations (2.7) and (2.8), we obtain the following small-time solutions for $\sigma_{r}$ and $\sigma_{\phi}$ :

$$
\begin{aligned}
& \sigma_{r}=\frac{x}{r} \sum_{\alpha=1}^{2}(-1)^{\alpha}\left\{\left(L_{\alpha}+N_{\alpha}\right)+\left[M_{\alpha}+P_{\alpha}+\frac{2}{r}(1-\eta) V_{\alpha} L_{\alpha}\right] \tau_{\alpha}\right\} H\left(\tau_{\alpha}\right), \\
& \sigma_{\phi}=\frac{x}{r} \sum_{\alpha=1}^{2}(-1)^{\alpha}\left\{\eta L_{\alpha}+N_{\alpha}+\left[\eta M_{\alpha}+P_{\alpha}+\frac{1}{r}(\eta-1) V_{\alpha} L_{\alpha}\right] \tau_{\alpha}\right\} H\left(\tau_{\alpha}\right) .
\end{aligned}
$$

The forms of the solutions (4.3), (4.5), and (4.6) confirm the existence of two coupled waves emanating from the boundary of the cavity and propagating outward with constant speeds $V_{\alpha}$, and having $\tau_{\alpha}=0$ as wavefronts. Bearing in mind that $V_{1}>V_{2}$, we check that these solutions are identically zero for $r>a+t V_{1}$. This means that the effects of the waves are localized at a given instant of time $t^{*}>0$, the points of the body that are beyond the faster wavefront $\left(r=a+V_{1} t^{*}\right)$ do not experience any disturbance. This observation verifies that the GN-theory is indeed a generalized thermoelasticity theory.

As mentioned earlier, the counterparts of our problem in the contexts of the conventional coupled thermoelasticity theory, the GL-theory and the LS-theory have been considered in $[16,18,17]$. In these works also, like in our analysis here, only small-time solutions have been derived. By comparing the solutions obtained in these works with 
our solutions, we find that whereas the solutions in the contexts of the three theories just mentioned decay exponentially with increasing $r$, our solutions do not exhibit such an exponential decay. This is due to the fact that the GN-theory does not admit dissipation of thermal energy. In this connection, it may be mentioned that in the context of the uncoupled thermoelasticity theory also, the exponential decay is not observed [20].

5. Analysis of discontinuities. By direct inspection of solutions (4.3), (4.5), and (4.6) we can determine the discontinuities experienced by the field functions across the wavefronts. These discontinuities are

$$
\begin{gathered}
{[u]_{\alpha}=0,} \\
{[\theta]_{\alpha}=\frac{x}{r}(-1)^{\alpha+1} N_{\alpha},} \\
{\left[\sigma_{r}\right]_{\alpha}=\frac{x}{r}(-1)^{\alpha}\left(L_{\alpha}+N_{\alpha}\right),} \\
{\left[\sigma_{\phi}\right]_{\alpha}=\frac{x}{r}(-1)^{\alpha}\left(\eta L_{\alpha}+N_{\alpha}\right) .}
\end{gathered}
$$

Here, $[\cdots]_{\alpha}$ denotes the discontinuity of the function across the wavefront $\tau_{\alpha}=0$.

It should be noted that, since the discontinuities in the field functions are precisely the coefficients of $H\left(\tau_{\alpha}\right)$ that do not contain $\tau_{\alpha}$ in the corresponding solutions, the discontinuities documented above are exact, although they are derived from smalltime solutions.

Expression (5.1) shows that the displacement is continuous across both the wavefronts. This is the situation in the context of the LS-theory also [16]. But in the contexts of the conventional coupled theory and the GL-theory, the displacement has been found to be discontinuous at the wavefronts [18]. A discontinuity in displacement implies that one portion of matter penetrates into another, and this phenomenon violates the continuum hypothesis [8]. Thus, as far as the behaviour of the displacement near the wavefronts is concerned, the predictions of the conventional coupled thermoelasticity theory and the GL-theory are physically absurd.

Expressions (5.2) show that the temperature and the radial and circumferential stresses are all discontinuous at both wavefronts and that the magnitudes of discontinuities are finite. The same situation arises in the context of the LS-theory also [16]. But the case with the GL-theory is different according to this theory, the stresses suffer delta function singularities at both wavefronts. This predictions of the GL-theory is also not physically realistic.

Thus, as far as the discontinuities across the wavefronts are concerned, the predictions of the GN-theory (as obtained here) are qualitatively similar to those of the LS-theory. Furthermore, like the LS-theory, the GN-theory also does not possess physically unrealistic features inherent in the conventional coupled thermoelasticity theory and the GL-theory (as described above).

\section{REFERENCES}

[1] M. A. Biot, Thermoelasticity and irreversible thermodynamics, J. Appl. Phys. 27 (1956), 240-253. MR 17,1035e. Zbl 071.41204. 
[2] D. S. Chandrasekharaiah, Thermoelasticity with second sound: A review, Appl. Mech. Rev. 39 (1986), 355-376 (English). Zbl 588.73006.

[3] _ A note on the uniqueness of solution in the linear theory of thermoelasticity without energy dissipation, J. Elasticity 43 (1996), no. 3, 279-283. MR 97e:73009. Zbl 876.73014.

[4] _ One-dimensional wave propagation in the linear theory of thermoelasticity without energy dissipation, J. Thermal Stresses 19 (1996), 695-712.

[5] _ Thermaelastic plane waves without energy dissipation, Mech. Res. Comm. 23 (1996), no. 5, 549-555. Zbl 895.73010.

[6]___ A uniqueness theorem in the theory of thermoelasticity without energy dissipation, J. Thermal Stresses 19 (1996), no. 3, 267-272. MR 97i:73009.

[7] _ Thermoelastic Reyleigh waves without energy dissipation, Mech. Res, Commns. 24 (1997), 93-102.

[8] D. S. Chandrasekharaiah and L. Debnath, Continuum Mechanics, Academic Press Inc., Boston, MA, 1994. MR 95h:73003. Zbl 798.73001.

[9] D. S. Chandrasekharaiah and K. S. Srinath, One-dimensional waves in a thermoelastic halfspace without energy dissipation, Internat. J. Engrg. Sci. 34 (1996), no. 13, 14471455. Zbl 900.73125.

[10] _ Axisymmetric thermoelastic interactions without energy dissipation in an unbounded body with cylindrical cavity, J. Elasticity 46 (1997), no. 1, 19-31. Zbl 886.73009.

[11]__ Thermalelastic plane waves without energy dissipation in a half-space due to timedependent heating of the boundary, J. Thermal Stresses 20 (1997), 659-676.

[12] _ Thermoelastic plane waves without energy dissipation in a rotating body, Mech. Res. Comm. 24 (1997), no. 5, 551-560. Zbl 896.73012.

[13] A. E. Green and K. A. Lindsay, Thermoelasticity, J. Elasticity 2 (1972), no. 1, 1-7. Zbl 775.73063.

[14] A. E. Green and P. M. Naghdi, Thermoelasticity without energy dissipation, J. Elasticity 31 (1993), no. 3, 189-208. MR 94f:73007. Zbl 784.73009.

[15] H. W. Lord and Y. A. Shulman, A generalized dynamical theory of thermoelasticity, J. Mech. Phys. Solids 15 (1967), 299-309. Zbl 156.22702.

[16] B. Mukhopadhyay, R. Bera, and L. Debnath, On generalized thermoelastic disturbances in an elastic solid with a spherical cavity, J. Appl. Math. Stochastic Anal. 4 (1991), no. 3, 225-240. Zbl 737.73030.

[17] G. A. Nariboli, Spherically symmetric thermal shock in a medium with thermal and elastic deformations coupled, Quart. J. Mech. Appl. Math. 14 (1961), 75-84. MR 22\#12900. Zbl 096.39204.

[18] S. K. Roychoudhuri and G. Chatterjee, Spherically symmetric thermoelastic waves in a temperature-rate-dependent medium with spherical cavity, Comput. Math. Appl. 20 (1990), 1-12.

[19] I. N. Sneddon, The Use of Integral Transforms, McGraw-Hill, New York, 1972, Chap. 3.

[20] E. Sternberg and J. G. Chakravorty, Thermal shock in an elastic body with a spherical cavity, Quart. Appl. Math. 17 (1959), 205-218. MR 21\#6149.

Chandrasekharaiah: Department of Mathematics, Bangalore University, Central COLLEGE CAMPUS, BANGALORE 560 001, INDIA

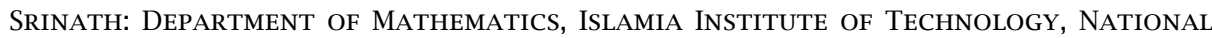
PARK ROAD, BANGALORE 560 076, INDIA 


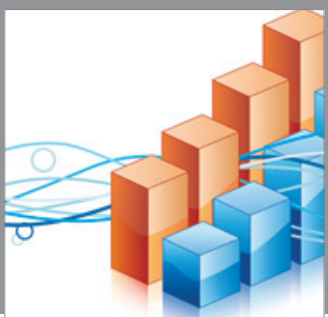

Advances in

Operations Research

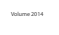

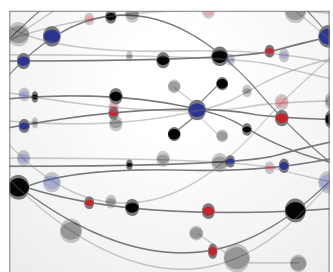

\section{The Scientific} World Journal
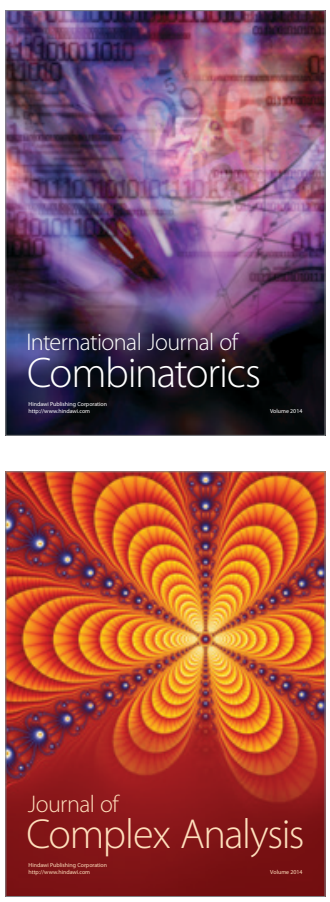

International Journal of

Mathematics and

Mathematical

Sciences
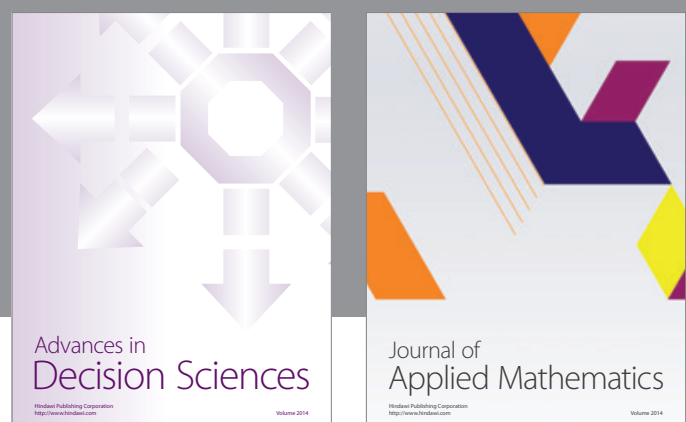

Journal of

Applied Mathematics
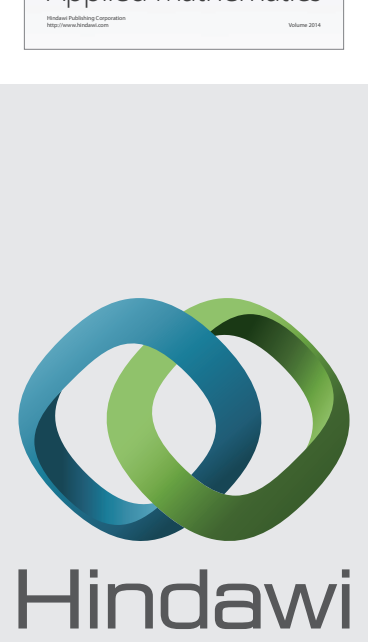

Submit your manuscripts at http://www.hindawi.com
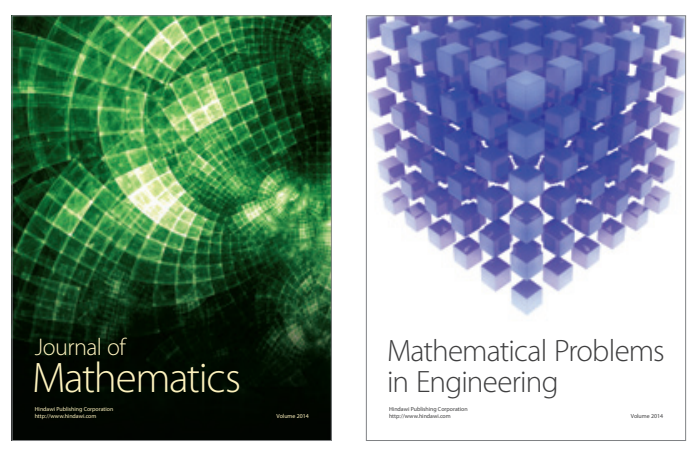

Mathematical Problems in Engineering
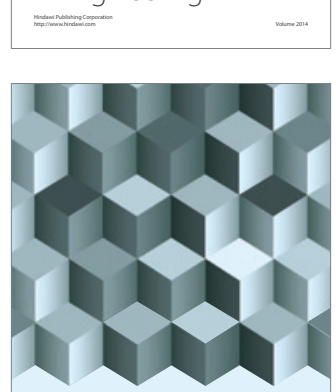

Journal of

Function Spaces
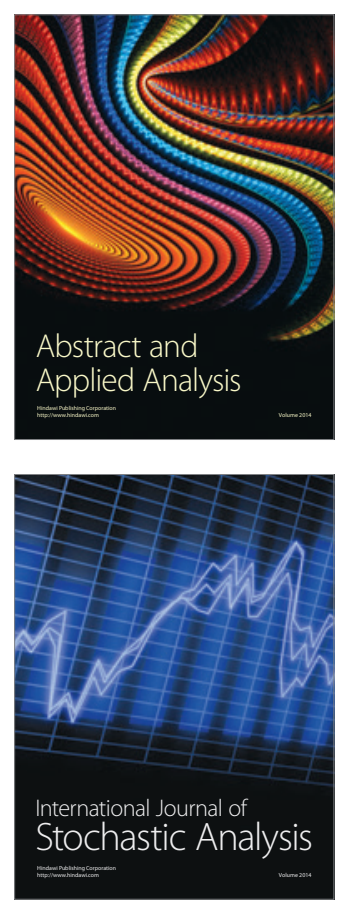

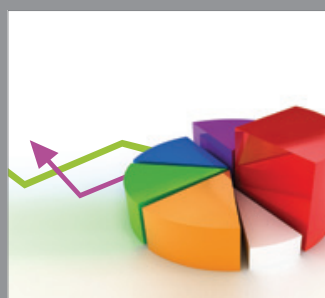

ournal of

Probability and Statistics

Promensencen
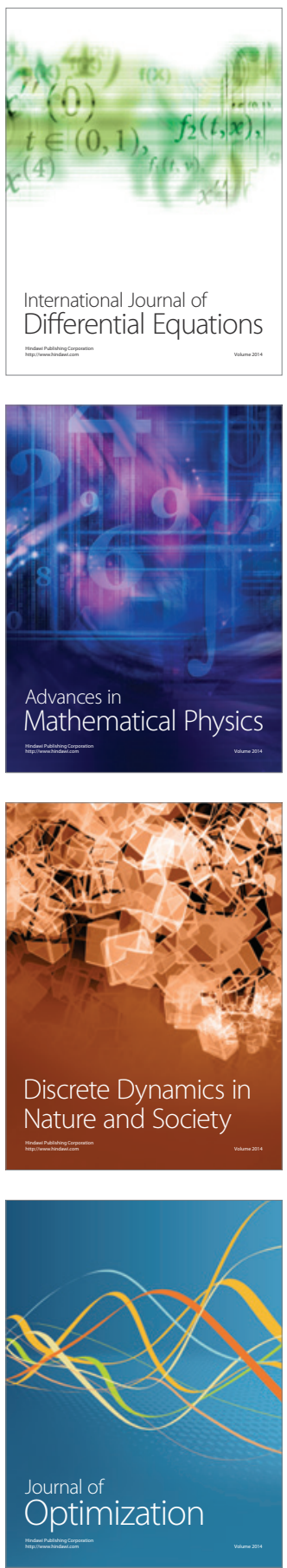